\title{
NEW CONCEPTS FOR QUASI-OPTICAL STRUCTURES FOR USE WITH GYROTRON SYSTEMS
}

\author{
M. Petelin ${ }^{1}$, Member IEEE, V. Erckmann ${ }^{2}$, J.L. Hirshfield ${ }^{3}$, W. Kasparek ${ }^{4}$, D. Shchegolkov ${ }^{5}$, A. Tolkachev ${ }^{6}$
}

\begin{abstract}
This paper discusses the use of some relatively new quasi-optical structures for configuring high-power gyrotronbased millimeter-wave systems for plasma fusion, microwave technologies, electron accelerators, radar and communication. Techniques for wave combining and switching, and for wave matching to transmitters, receivers and antennas are analyzed.
\end{abstract}

Index terms-- quasi-optics, wave dividers/combiners, multiplexers, duplexers, gyrotrons

\section{INTRODUCTION}

For reduction of RF losses and for minimizing the incidence of RF breakdown and RF fatigue in high-power millimeterwave systems, all components should have dimensions much larger than the RF wavelength. Accordingly, to maintain RF field coherence, efficient mode filtration methods should be used. For high-frequency gyrotron systems, this requirement is usually met by using quasi-optical transmission lines employing mirrors and/or by using oversized corrugated-wall waveguides [1], as illustrated in Fig.1. To match these quasioptical lines with RF sources and various loads, there is a need for appropriate mode converters, wave combiners, multiplexers, polarizers, directional couplers, rotary joints, filters, resonant rings, pulse compressors, isolators, etc. Some of these components and their combinations will be discussed in this paper. Portions of the material discussed here have been presented earlier [2], [3].

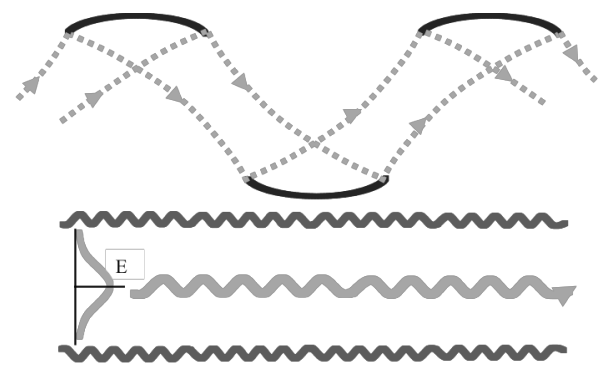

Figure 1. Quasi-Gaussian beam guides.

\footnotetext{
${ }^{1}$ IAP, Nizhny Novgorod, Russia, petelin@appl.sci-nnov.ru

2 IPP, Greifswald, Germany

${ }^{3}$ Yale University, New Haven, CT, USA

${ }^{4}$ Stuttgart University, Stuttgart, Germany

${ }^{5}$ IAP, Nizhny Novgorod, Russia

${ }^{6}$ JSC "Radio physics", Moscow, Russia
}

\section{COHERENT WAVE-BEAM COMBINERS}

It is known [4] that the transverse spatial distributions of quasi-optical beams can be transformed within a rather broad class of configurations by sequences of curved mirrors. For instance, Fig.2a illustrates an arrangement for dividing a primary beam into a number of secondary beams. The same function can be performed with diffraction gratings, as illustrated in Fig.2b [5]. If the grating period corresponds to splitting the primary beam into $n$ secondary beams, the RF power distribution between the secondary beams is governed by $n$ "essential" parameters in the grating profile; in particular, the power in each of the secondary beams can be equalized [6]. In both cases shown in Fig.2, the optical beam dividers also work reciprocally as beam combiners if the wave propagation directions are reversed and the beams being combined are properly phased-locked.

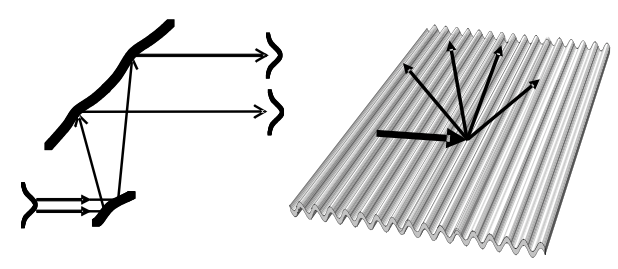

Figure 2. Quasi-optical dividers: a) curved-mirrors and b) diffraction grating. Reversing the beam propagation direction converts each device into an RF-beam combiner.

As an example, an arrangement similar to that shown in Fig. $2 \mathrm{a}$ can be used to match a system of duplexers (see section 4) to a monostatic monopulse radar antenna [7], [8] (Fig.3). In fact, matching of duplexers with transmit and receive units can be provided by either of the arrangements shown in Fig.2.

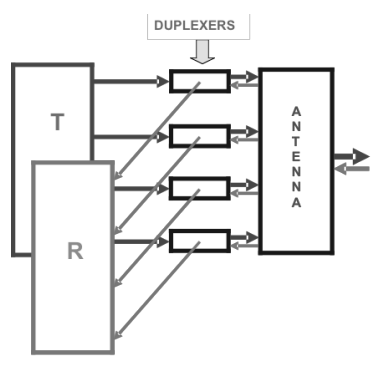

Figure 3. A radar block diagram of monopulse matching of antenna to transmitter $(\mathrm{T})$ and receiver $(\mathrm{R})$. 
A specific means to control RF beams can be provided with gratings of the Littrow configuration when the incident beam and the $-1^{\text {st }}$ order scattered beam share a common plane with the groove direction [5]. With a proper corrugation depth, a grating based on this configuration can function as a four-port $-3 \mathrm{~dB}$ hybrid (analogous to the usual magic T) [9]. When two RF beams of equal power are combined, the combined beam direction is switched from one output to another by changing the mutual phase-shift between the input beams. Fig.4 illustrates this concept and Fig.5 shows an experimental setup to confirm the idea, with supporting data. Magic Y's (as this grating arrangement is dubbed) might be proposed, in particular, for a delay line distribution system of a future linear electron accelerator designed to operate at sufficiently high carrier frequency [10], [11]. Fig.6 illustrates this application.

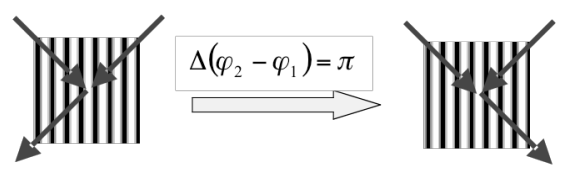

Figure 4. Phase-controlled quasi-optical 3 dB hybrid: Magic Y.

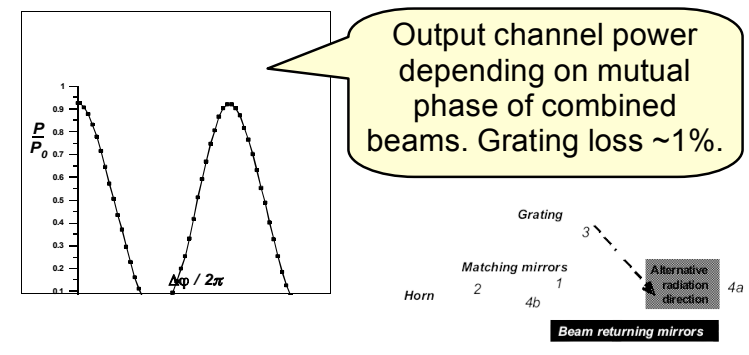

Figure 5. A photo and performance of a $34 \mathrm{GHz}$ magic $\mathrm{Y}$.

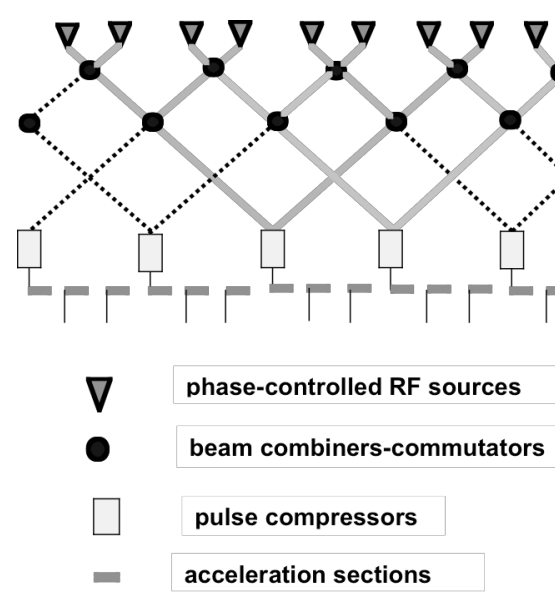

Figure 6. A scheme for a quasi-optical phased array to feed linear electron accelerator.

The above RF beam combining/switching techniques may also be used in plasma heating and current drive systems at large fusion test facilities, such as tokamaks and stellarators [12], [13]. When controlled by a plasma displacement signal, the structure illustrated in Fig.7 can be used to suppress hydrodynamic plasma instabilities $[14,15]$.

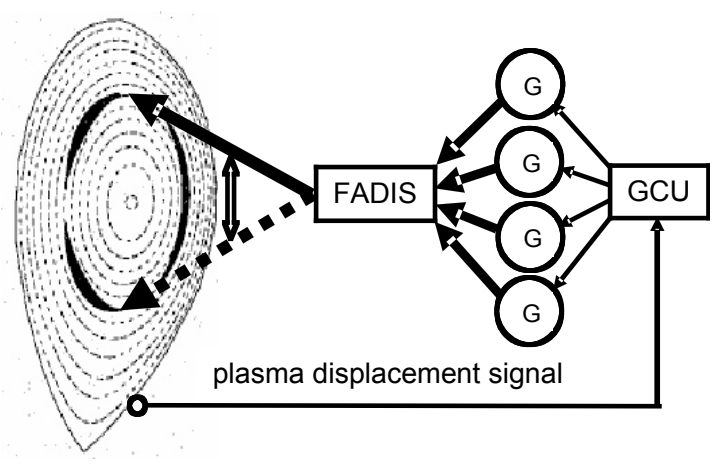

Figure 7. FADIS - fast adaptive discrete RF beam switch to suppress plasma instability: $\mathrm{G}$ - gyrotrons, GCU - gyrotron control unit.

All of the above-mentioned approaches imply the use of high-power RF amplifiers driven with a common lowpower master oscillator. In the millimeter-wave band, the highest-power amplifiers are presently of the gyrotron type, including gyroklystrons, gyrotwystrons and gyroTWTs. However, at present, these amplifiers have average and CW powers considerably less than what can be delivered by free-running gyrotron oscillators. Oscillators can also be used in techniques similar to that shown in Fig.7, but in this case beam combining and switching must be performed by multiplexing, as discussed below.

\section{MULTIPLEXERS}

The most popular kind of multiplexer is the dielectric prism; which splits a multi-frequency beam into a set of narrowfrequency beams and, reciprocally, combines a set of narrowfrequency beams into a single multi-frequency beam. Regretfully, at millimeter-waves frequency the dielectric prism cannot withstand high powers and must be replaced with an equivalent metallic structure.

If frequency bandwidths are in the range 1 to $10 \%$, multiplexing can be performed with a grating of the Littrow type, as shown in Fig.2b. In this case, some power is lost due to specular reflection [5], [6]. Such multiplexers can be used in radar and communication systems with synthesized frequency bands, as illustrated in Fig. 8 [9], [16], [17]. 


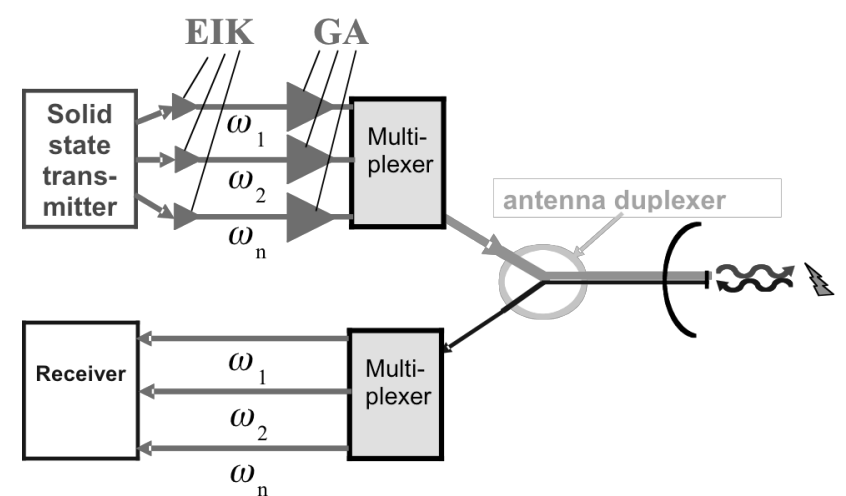

Figure 8. A block diagram for a millimeter-wave radar with a synthesized frequency band: EIK - extended interaction klystron [18], GA - gyroamplifier.

If frequency band widths are narrower, not exceeding $1 \%$, multiplexers of resonant and interferometric types seem preferable [9], [14-16]. An example of this approach is a ring mirror cavity coupled to input and output wave flows by a mirror corrugation, as illustrated in Fig. 9.

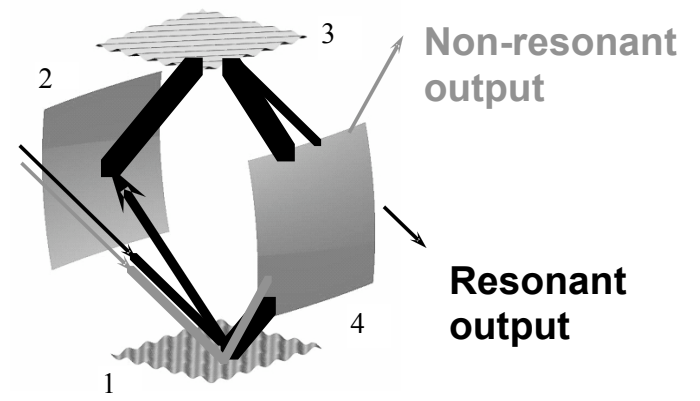

Figure 9. Scheme for a quasi-optical resonant diplexer: 1 and 3 are input and output gratings, 2 and 4 are phase-correcting mirrors.

For this diplexer, if the input wave is non-resonant, the wave is reflected from the input grating 1 into the specular direction; whereas if the input wave is resonant with the cavity, the cavity is excited and the RF power is radiated by the grating 3 into the $-1^{\text {st }}$ order diffraction beam direction. Due to the up-down symmetry of the resonant diplexer shown in Fig.9, it can be fed through the grating 3 simultaneously with grating 1 . With a proper mutual frequency shift, RF power from the both input beams can be directed into either of the common output channels. By commutation of the incident frequencies, the combined beam can be switched from one output channel to another. Such a diplexer (Fig.10) [15] was used to combine the power from two near-140 $\mathrm{GHz}$ sub-MW gyrotrons with voltage-controlled frequencies; the combined power was switched between two output channels at switching frequencies up to $\sim 10 \mathrm{kHz}$, which is typical for the neoclassical tearing mode (NTM) instability in modern tokamaks.

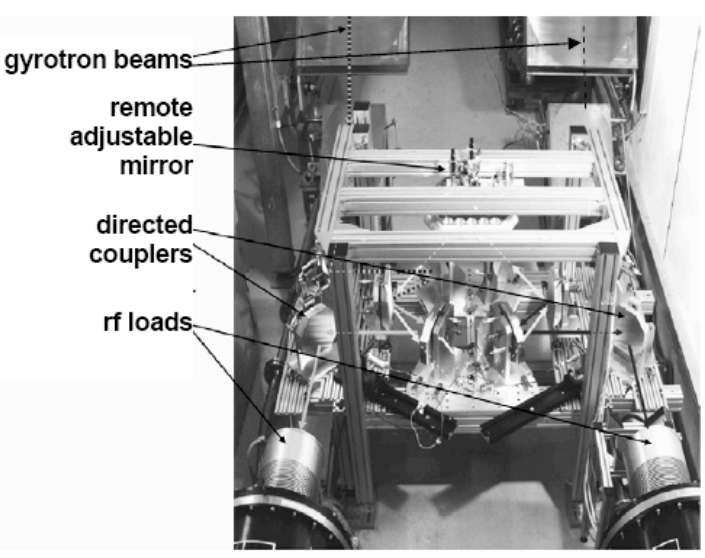

Figure 10. A diplexer combining RF beams from two gyrotrons and switching the combined $\sim 1 \mathrm{MW} / 140 \mathrm{GHz}$ beam between two output channels with $\sim 10 \mathrm{kHz}$ frequency.

Quasi-optical diplexers of both resonant and interferometric types could be configured into multiplexers with a relatively large number of inputs and outputs [9], [14-16]. In particular, multiplexers can provide the wave combiningswitching performance shown in Fig.7, when gyrotron voltages are modulated and, accordingly, gyrotron frequencies are varied [14], [15]. In tokamaks, in addition to plasma heating and current drive, multiplexers can be used for plasma diagnostics, in particular, for sub- $\mathrm{THz}$ reflectometry of dense near-diverter plasmas [19].

\section{DUPLEXERS}

A number of systems, in particular, those used in radar and communication, as in Fig. 3 and Fig. 8, need transmit-receive switches (duplexers) [7], [8], [2], [17] in order to forward the radiation from the transmitter to the antenna, and to direct the receive signal from the antenna to the receiver. In this application, direct penetration of RF power from the transmitter to the receiver should be minimized.

To withstand high powers, millimeter-wave duplexers should be made of oversized metallic elements. In particular, if transmit-receive RF beams are circularly polarized, the duplexer can be composed of two successive gratings functioning respectively as a polarization separator and a polarizer [9]. This concept is illustrated in Fig.11. The simplest version built of such a duplexer [20] had a frequency bandwidth of about $2 \%$, limited by the frequency scan of the $-1^{\text {st }}$ order diffraction ray at the polarization separator. When the scan was compensated by insertion of an additional dispersive Littrow grating (see Fig.12), the frequency band width was expanded to $8 \%$. The transmitreceive isolation was $45 \mathrm{~dB}$. 


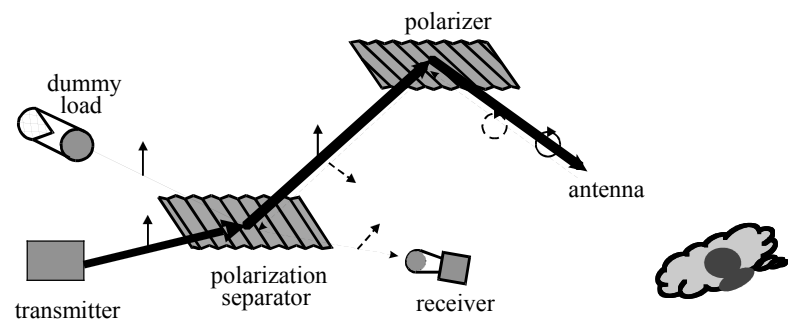

Figure 11. A scheme of grating-based duplexer.

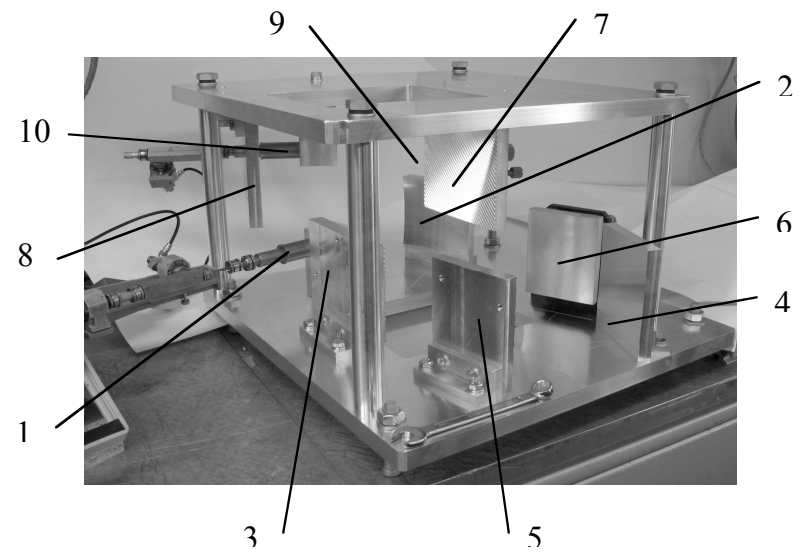

Figure 12. A model of a broad-band duplexer with a central frequency of $94 \mathrm{GHz}: 1$ and 10 are input and output horns; 3, 4 and 7 are gratings; 2, 5, 6, 8 and 9 are matching mirrors.

\section{CONCLUSION}

Quasi-optical systems can be convenient, flexible and efficient for directing, combining, and splitting high-power millimeter-wave RF beams. In addition to the examples given in this paper, such systems are also applied in microwave material processing, non-equilibrium plasma chemistry and production of multi-charged ions.

\section{ACKNOWLEDGEMENTS}

The authors are grateful to Alex Bruschi, George Caryotakis, Baruch Levush, Alexander Litvak, Gregory Nusinovich, Sami Tantawi and Manfred Thumm for encouraging discussions and helpful advice.

\section{REFERENCES}

[1] M. Thumm, W. Kasparek, Passive High-Power Microwave Components, IEEE Trans. PS, 30, No. 3, 2002, pp. 755-786.

[2] M. Petelin, M. Salin, D. Schegolkov et al., New Elements for Controlled Gyrotron Systems, Abstracts of IVEC 2008, Monterey, pp. 87-88.

[3] A. Tolkachev, B. Levitan, M. Petelin, Prospects of High Power Millimeter-Wave Radar, Abstracts of $7^{\text {th }}$ Int. Workshop "Strong Microwaves: Sources and Applications", IAP, Nizhny Novgorod, 2008, p. 132.

[4] V. A. Borovikov, B. E. Kinber, Geometrical Theory of Diffraction, Moscow, Svyaz, 1978.

[5] R. Petit (ed.), Electromagnetic theory of gratings, SpringerVerlag, 1980.

[6] E. Koposova, M. Petelin, Scattering of wave by metallic corrugated surface: control of distribution of scattered wave powers with profile optimization, Radio Phys. Quant. Electron, 32, No. 9, pp. 1178-1181, 1989.

[7] M. Skolnik, Introduction to Radar Systems, McGraw-Hill, 2001.

[8] D. Barton, Modern Radar System Analysis, Artech House, 1988.

[9] M. Petelin, G. Caryotakis, A. Tolkachev et al., Quasi-Optical Components for MMW Fed Radars and Particle Accelerators, in AIP Conference Proc. 474, Ed. R. Phillips, Woodbury, New York, pp. 304-315, 1998.

[10] M. Petelin, Quasi-Optical Collider Concept, Proc. of AAC-2002, CA, June 20, 2002.

[11] M. Petelin, V. Pavelyev, A. Yunakovsky et al., Components for quasi-optically fed linear accelerators, AIP Conf. Proc. 807, Ed. D. Abe and G. Nusinovich, 2005, pp. 408-415.

[12] R. Olstad, R. Callis, J. Doane et al., ECR wave transmission and control structures, ibid. [2], p. 76.

[13] M. Henderson, C. Darbos, F. Abajar et al., Overview of the ITER ECH\&CD system, ibid. [2], p. 69.

[14] W. Kasparek, M. Petelin, V. Erckmann et al., Fast switching and power combination of high-power electron cyclotron wave beams: principles, numerical results, and experiments, Fusion Science and Technology, 52, No. 2, 281-290, 2007.

[15] W. Kasparek, M. Petelin, D. Shchegolkov et al., A fast switch, combiner and narrow-band filter for high-power millimetre wave beams, Nucl. Fusion 48 054010, 2008, pp. 1-9.

[16] M. Petelin, G. Caryotakis, Yu. Postoenko et al., Quasi-optical multiplexers for space communication and radar with synthesized frequency band, in Quasi-optical control of intense microwave transmission, Ed. J. Hirshfield and M. Petelin, NATO Science Series 2, Mathematics, Physics and Chemistry, Vol. 203, Springer, 2005, pp. 185-198.

[17] M. Kodis, D. Abraham, D. Morabito, Deep space high power uplinks, AIP Conf. Proc. 691, Ed. S. Gold and G. Nusinovich, 2003 , pp. 47-53.

[18] B. Steer, A. Roitman, P. Horoyski et al., Millimeter Wave Amplifiers of Medium Power, ibid. [2], p. 133.

[19] N. Skvortsova, G. Batanov, K. Sarksyan et al., Opportunities for plasma diagnostics in fusion devices by means of terahertz sources, ibid. [2], p. 90.

[20] J.L. Hirshfield, P. Kolchin, S. Kuzikov, M. Petelin, Quasi-optical antenna duplexer, Digest $25^{\text {th }}$ Conf. on IR\&MMW, Beijing, 405-406, 2000 . 